\title{
The Homological Kähler-de Rham Differential Mechanism: II. Sheaf-Theoretic Localization of Quantum Dynamics
}

\author{
Anastasios Mallios and Elias Zafiris \\ Department of Mathematics, University of Athens, Panepistimioupolis, 15784 Athens, Greece \\ Correspondence should be addressed to Elias Zafiris, ezafiris@math.uoa.gr
}

Received 7 March 2011; Accepted 30 May 2011

Academic Editor: Mauro Francaviglia

Copyright (C) 2011 A. Mallios and E. Zafiris. This is an open access article distributed under the Creative Commons Attribution License, which permits unrestricted use, distribution, and reproduction in any medium, provided the original work is properly cited.

The homological Kähler-de Rham differential mechanism models the dynamical behavior of physical fields by purely algebraic means and independently of any background manifold substratum. This is of particular importance for the formulation of dynamics in the quantum regime, where the adherence to such a fixed substratum is problematic. In this context, we show that the functorial formulation of the Kähler-de Rham differential mechanism in categories of sheaves of commutative algebras, instantiating generalized localization environments of physical observables, induces a consistent functorial framework of dynamics in the quantum regime.

\section{Introduction}

The basic conceptual and technical issue pertaining to the current research attempts towards the construction of a viable quantum theory of the gravitational field, refers to the problem of independence of this theory from a fixed spacetime manifold substratum. In relation to this problem, we have argued about the existence and functionality of a homological schema of functorial general relativistic dynamics, constructed by means of connection inducing functors and their associated curvatures, which is, remarkably, independent of any background substratum [1]. More precisely, the homological dynamical mechanism is based on the modeling of the notion of physical fields in terms of connections, which effectuate the functorial algebraic process of infinitesimal scalars extensions, due to interactions caused by these fields. The appealing property of this schema lies on the fact that the induced field dynamics is not dependent on the codomain of representability of the observables and most importantly is only subordinate to the algebra-theoretic characterization of their structures. In this perspective, the absolute representability principle of classical general relativity, in terms of real numbers, may be relativized without affecting the functionality of the algebraic 
mechanism. Consequently, it is possible to describe the dynamics of gravitational interactions in generalized localization environments, instantiated by suitable categories of presheaves or sheaves. In particular, according to this strategy, the problem of quantization of gravity is equivalent to forcing the algebraic Kähler-de Rham general relativistic dynamical mechanism of the gravitational connection functorial morphism inside an appropriate sheaf-theoretic localization environment, which is capable of incorporating the localization properties of observables in the quantum regime. The only cost to be paid for this sheaf-theoretic localization [2] is the rejection of the fixed background manifold structure of the classical theory. This is actually not a cost at all, since it would permit the intelligibility of the field equations over geometric realizations that include manifold singularities and other pathologies, without affecting the algebraic mechanism of dynamics, like that in $[3,4]$. We argue that the Kähler-de Rham general relativistic dynamical mechanism can be localized in the quantum regime of observable structure by incorporating the novel conception of physical localization associated with the notion of a Grothendieck topos [5, 6]. In particular, the crucial observation that makes the Grothendieck topos theoretic environment relevant is that a globally noncommutative or even partially commutative algebra of quantum observables determines an underlying categorical diagram (presheaf) of commutative observable algebras. Then, each one of the latter can be locally identified, in a precise sheaftheoretic way that makes use of the notion of an appropriate Grothendieck topology, with a commutative algebra of classical observables. Consequently, the physical information contained in a quantum observable algebra can be recovered by a gluing construction referring to its local commutative subalgebras [7-12].

\section{Topological Sheaf-Theoretic Dynamics via Abstract Differential Geometry}

The absolute representability principle of the classical general theory of relativity is based on the set-theoretic conception of the real line, as a set of infinitely distinguished points coordinatized by means of the field of real numbers. Expressed categorically, this is equivalent to the interpretation of the algebraic structure of the reals inside the absolute universe of Sets, or more precisely inside the topos of constant Sets. It is also well known that algebraic structures and mechanisms can admit a variable reference, formulated in category-theoretic jargon in terms of arrows only specifications, inside any suitable topos of discourse [6,13]. A general topos can be conceived as a manifestation of a universe of variable sets. For example, the topos of sheaves of sets $\operatorname{Shv}(X)$ over the category of open sets of an abstract topological space $X$, ordered by inclusion, is understood as a categorical universe of varying sets over the open reference domains of the topology covering $X$. The relativization of physical representability, with respect to the topos of sheaves $\operatorname{Shv}(X)$, amounts to the relativization of both the notion and the algebraic structure of the real numbers inside this topos [7]. Regarding the notion of real numbers inside the topos $\operatorname{Shv}(X)$, this is equivalent to the notion of continuously variable real numbers over the open domains of $X$, or else, equivalent to the notion of real-valued continuous functions on $X$, when interpreted, respectively, inside the topos of Sets $[6,7,13]$. Regarding the algebraic structure of the reals inside the topos $\operatorname{Shv}(X)$, they form only an algebra in this topos, which is identical with the sheaf of $\mathbf{R}$-algebras of continuous real-valued functions on $X$, where $\mathbf{R}$ corresponds in that case to the constant sheaf of real numbers over $X$.

According to the functorial Kähler-de Rham general relativistic dynamical mechanism [1], the absolute representability principle of classical General Relativity, in terms of real 
numbers, may be relativized locally with respect to a category of measurement loci without affecting the functionality of the physical dynamical mechanism. Consequently, it is possible to describe the dynamics of gravitational interactions in generalized localization environments. The latter are understood in the sense of categories of presheaves, defined over a base category of reference loci, or categories of sheaves with respect to some suitable topological covering system.

From a physical viewpoint, the construction of a sheaf of algebras of observables constitutes the natural outcome of a complete localization process [2, 7]. Generally speaking, a localization process is being implemented in terms of an action of some category of reference contexts on a set-theoretic global algebra of observables. The latter, is then partitioned into sorts parameterized by the objects of the category of contexts. In this way, the functioning of a localization process can be represented by means of a fibered construct, understood geometrically as a presheaf or, equivalently, as a variable set (algebra) over the base category of contexts. The fibers of this presheaf may be thought, in analogy to the case of the action of a group on a set of points, as the "generalized orbits" of the action of the category of contexts. The notion of functional dependence, incorporated in this action, forces the global algebraic structure of observables to fiber over the base category of reference contexts, giving rise to a presheaf of algebras of observables. According to the physical requirements of observability, for every reference context of the base category, the set of local observables defined over it constitutes a commutative unital algebra over the real numbers. The transition from a presheaf to a sheaf of algebras of observables requires the following: firstly, the concrete specification of a topological covering system, on the category of reference contexts, interpreted as a localization scheme of the global algebraic structure of observables. Secondly, the collation of local observable information into global ones effectuated via a compatible family of local sections of the presheaf over a localization system. If a locally compatible choice of observables induces a unique global choice, then the condition for being a sheaf is satisfied. We note that, in general, there will be more locally defined or partial choices than globally defined ones, since not all partial choices need be extendible to global ones, but a compatible family of partial choices uniquely extends to a global one; or in other words, any presheaf uniquely defines a sheaf.

In the sequel, we consider the localization environment of the category of sheaves of sets $\operatorname{Shv}(X)$ defined over the category of open sets $\mathcal{O}(X)$ of an abstract topological space $X$, ordered by inclusion. We define a topological covering system in the environment of $\mathcal{O}(\mathcal{X})$ as follows.

A topological covering system on $\mathcal{O}(\mathcal{X})$ is an operation $J$, which assigns to each open reference domain $U$ in $\mathcal{O}(\mathcal{X})$, a collection $J(U)$ of $U$-sieves called topological covering $U$ sieves, such that the following three conditions are satisfied.

(1) For every open reference domain $U$ in $\mathcal{O}(\mathcal{X})$, the maximal sieve $\{g: \operatorname{cod}(g)=U\}$ belongs to $J(U)$ (maximality condition).

(2) If $S$ belongs to $J(U)$ and $h: V \rightarrow U$ is a figure of $U$, then $h^{*}(S)=\{f: V \rightarrow$ $U,(h \circ f) \in S\}$ belongs to $J(V)$ (stability condition).

(3) If $S$ belongs to $J(U)$ and if for each figure $h: V_{h} \rightarrow U$ in $S$ there is a sieve $R_{h}$ belonging to $J\left(V_{h}\right)$, then the set of all composites $h \circ g$, with $h \in S$ and $g \in R_{h}$, belongs to $J(U)$ (transitivity condition).

As a consequence of the conditions above, we can check that any two $U$-covering sieves have a common refinement; that is, if $S, R$ belong to $J(U)$, then $S \cap R$ belongs to $J(U)$. 
If we consider the partially ordered set of open subsets of a topological measurement space $X$, viewed as the category of base reference loci $\mathcal{O}(\mathcal{X})$, then we specify that $S$ is a covering $U$-sieve if and only if $U$ is contained in the union of open sets in $S$. The above specification fulfills the requirements of topological covering sieves posed above and consequently, defines a topological covering system on $\mathcal{O}(\mathcal{X})$. From a physical perspective, the consideration of covering sieves as generalized measures of localization of observables, within a global observable structure, gives rise to localization systems of the latter.

Furthermore, we can show that if $\mathbb{A}$ is the contravariant presheaf functor that assigns to each open set $U \subset X$, the commutative unital algebra of all continuous observables on $U$ then $\mathbb{A}$ is actually a sheaf of sets. This is intuitively clear since the specification of a topology on a measurement space $X$ (and, hence, of a topological covering system on $\mathcal{O}(\mathcal{X})$ as previously) is solely used for the definition of the continuous observables on $X$, and thus, the continuity of each observable can be determined locally. This means that continuity respects the operation of restriction to open sets and moreover that continuous observables can be collated in a unique manner, as it is required for the satisfaction of the sheaf condition. More precisely, the sheaf condition means that the following sequence of commutative unital $\mathbb{R}$ algebras of local observables is left exact:

$$
0 \longrightarrow \mathbb{A}(U) \longrightarrow \prod_{a} \mathbb{A}\left(U_{a}\right) \longrightarrow \prod_{a, b} \mathbb{A}\left(U_{a b}\right)
$$

It is instructive to emphasize that the algebraic functorial Kähler-de Rham framework of dynamics is based for its conceptualization and operative efficacy, neither on the methodology of real analysis nor on the restrictive assumption of smoothness of observables, but only, on the functorial expression of the inverse processes of infinitesimal scalars extension/restriction. Nevertheless, it is instructive to apply this algebraic framework for the case of algebra sheaves of smooth observables, in order to reproduce the differential geometric mechanism of smooth manifolds geometric spectra, interpreted in the localization environment of the category $\operatorname{Shv}(X)$. For this purpose, we consider that $\mathbb{A}$ stands for the sheaf of algebras of $\mathbb{R}$-valued smooth functions on $X$, denoted by $\mathcal{C}^{\infty}$, whereas $\Omega^{n}(\mathbb{A})$ stand in this context for the locally free sheaves of $\mathcal{C}^{\infty}$-modules of differential $n$-forms on $X$. In this case, the algebraic de Rham complex of $\mathbb{A}$, gives rise to the corresponding differential de Rham complex of $\mathcal{C}^{\infty}$, as follows:

$$
\mathcal{C}^{\infty} \longrightarrow \Omega^{1}\left(\mathcal{C}^{\infty}\right) \longrightarrow \cdots \longrightarrow \Omega^{n}\left(\mathcal{C}^{\infty}\right) \longrightarrow \cdots
$$

The crucial mathematical observation concerning this complex, refers to the fact that, the augmented differential de Rham complex

$$
0 \longrightarrow \mathbb{R} \longrightarrow \mathcal{C}^{\infty} \longrightarrow \Omega^{1}\left(\mathcal{C}^{\infty}\right) \longrightarrow \cdots \longrightarrow \Omega^{n}\left(\mathcal{C}^{\infty}\right) \longrightarrow \cdots
$$

is actually exact. The exactness of the augmented differential de Rham complex, as above, constitutes an expression of the lemma of Poincaré, according to which every closed $\mathcal{C}^{\infty}$ form on $X$ is exact at least locally in $X$. Thus, the welldefinability of the differential geometric dynamical mechanism of smooth manifolds is precisely due to the exactness of the augmented differential de Rham complex. This mathematical observation for the case 
of algebra sheaves of smooth observable coefficients, raises the issue of enrichment of the general functorial mechanism of infinitesimal scalars extensions, by the requirement of exactness of the respective augmented algebraic de Rham complex, securing in this sense the well-definability of the dynamical mechanism for the general case and reproducing the corresponding differential geometric mechanism of smooth manifolds faithfully, as well. This requirement implements precisely the principle of covariance of dynamics with respect to cohomologically well-behaved algebras of physical observables, interpreted within the localization environment of $\operatorname{Shv}(X)$.

A complete settlement of this issue, addressing the principle of covariance as above, comes from the mathematical theory of the abstract differential geometry (ADG) [14-16]. Actually, the axiomatic development of (ADG), in a fully-fledged mathematical theory, has been based on the exploitation of the consequences of the above-stated mathematical observation for the case of algebra sheaves of smooth observable coefficients. In this sense, the operational machinery of (ADG) is essentially implemented by the imposition of the exactness requirement of the following abstract de Rham complex, interpreted inside the topos of sheaves $\operatorname{Shv}(X)$ :

$$
0 \longrightarrow \mathbb{R} \longrightarrow \mathbb{A} \longrightarrow \Omega^{1}(\mathbb{A}) \longrightarrow \cdots \longrightarrow \Omega^{n}(\mathbb{A}) \longrightarrow \cdots
$$

ADG's power of abstracting and generalizing the classical differential calculus on smooth manifolds basically lies on the possibility of assuming other more general coordinate sheaves $\mathbb{A}$, while at the same time retaining, via the exactness of the algebraic augmented de Rham complex as above, the mechanism of differentials instantiated paradigmatically, in the first place, in the case of classical differential geometry on smooth manifolds.

For our physical purposes, we conclude that any cohomologically appropriate sheaf of algebras $\mathbb{A}$, characterized by the exactness property posed previously, can be legitimately regarded as a sheaf of local observables, capable of providing a well-defined dynamical mechanism, independently of any smooth manifold background, analogous, however, to the one supported by smooth manifolds.

Conclusively, it is instructive to recapitulate and add some further remarks on the physical semantics associated with the preceding algebraic cohomological dynamical framework by invoking the sheaf-theoretic terminology explicitly. The basic mathematical objects involved in the development of that framework consists of a sheaf of commutative unital algebras $\mathbb{A}$, identified as a sheaf of algebras of local observables, a sheaf of locally free $\mathbb{A}$ modules $E$ of rank $n$, as well as the sheaf of locally free $\mathbb{A}$-modules of universal 1 -forms $\Omega$ of rank $n$. We assume that these sheaves have a common base space, over which they are localized, namely, an arbitrary topological measurement space $X$. A topological covering system of $X$ is defined simply by an open covering $U=\{U \subseteq X: U$ open in $X\}$ of $X$ such that any locally free $\mathbb{A}$-module sheaf $N$ splits locally, by definition, that is, with respect to every $U$ in $\mathcal{U}$ into a finite $n$-fold Whitney sum $\mathbb{A}^{n}$ of $\mathbb{A}$ with itself as $\left.N\right|_{U}=\left.\mathbb{A}^{n}\right|_{U}$. For this reason, a topological covering system $\mathcal{U}$ of $X$ may be called a coordinatizing open cover of $\mathbf{N}$. Hence, the local sections of the structure $\mathbb{R}$-algebra sheaf $\mathbb{A}$ relative to the coordinatizing open cover $u$ obtain the meaning of local coordinates, while $\mathbb{A}$ itself may be called "the coefficient" or "continuously variable real number coordinate sheaf" of $N$.

At a further stage of development, the implementation of the notion of functorial dynamical connectivity requires the functorial modeling of the notion of a physical field in terms of a connection expressing the algebraic process of infinitesimal scalar extensions 
of the algebra sheaf of local observables. Thus, we conclude that a pair $\left(E, \nabla_{E}\right)$, consisting of a left $\mathbb{A}$-module sheaf $E$ and a connection $\nabla_{E}$ on $E$, represents a local causal agent of a variable interaction geometry, namely, a physical field acting locally and causing infinitesimal variations of coordinates, standing for local observables. In this sense, the local sections of $\mathbb{A}$ module sheaf $E$, relative to the open cover $U$, coordinatize the states of the corresponding physical field. The connection $\nabla_{E}$ on $E$ is given by an $\mathbb{R}$-linear morphism of $\mathbb{A}$-modules sheaves:

$$
\nabla_{E}: E \longrightarrow \Omega^{1}(\mathbb{A}) \bigotimes_{\mathbb{A}} E=E \bigotimes_{\mathbb{A}} \Omega^{1}(\mathbb{A}):=\Omega^{1}(E)
$$

such that the following Leibniz condition holds:

$$
\nabla_{E}(f \cdot v)=f \cdot \nabla_{E}(v)+d f \otimes v
$$

for all $f \in \mathbb{A}, v \in E$. Notice that, by definition, the connection $\nabla_{E}$ is only an $\mathbb{R}$-linear morphism of $\mathbb{A}$-modules sheaves. Hence, although it is $\mathbb{R}$-covariant, it is not $\mathbb{A}$-covariant as well. The connection $\nabla_{E}$ on $E$ contains the irreducible amount of information encoded in the process of infinitesimal scalars extension caused by local interactions, induced by the corresponding field.

A significant observation has to do with the fact that if $\mathbf{E}=\mathbb{A}$ considered as an $\mathbb{A}$ module over itself, then, the $\mathbb{R}$-linear morphism of sheaves of $\mathbb{A}$-modules

$$
d: \Omega^{0}(\mathbb{A}):=\mathbb{A} \longrightarrow \Omega(\mathbb{A})^{1}:=\Omega(\mathbb{A})
$$

is a natural connection, which is also integrable or flat, since $\langle\Omega(\mathbb{A})\rangle$ is actually a complex, namely, the algebraic de Rham complex of $\mathbb{A}$.

If we consider a coordinatizing open cover $e^{U} \equiv\left\{U ;\left(e_{i}\right)_{0 \leq i \leq n-1}\right\}$ of the $\mathbb{A}$-module sheaf $E$ of rank $n$, every continuous local section $s \in E(U)$, where $U \in \mathcal{U}$ can be expressed uniquely as a superposition

$$
s=\sum_{i=1}^{n} s_{i} e_{i}
$$

with coefficients $s_{i}$ in $\mathbb{A}(U)$. The action of $\nabla_{E}$ on these sections of $E$ is expressed as follows:

$$
\nabla_{E}(s)=\sum_{i=1}^{n}\left(s_{i} \nabla_{E}\left(e_{i}\right)+e_{i} \otimes d\left(s_{i}\right)\right),
$$

where

$$
\nabla_{E}\left(e_{i}\right)=\sum_{i=1}^{n} e_{i} \otimes \omega_{i j}, \quad 1 \leq i, j \leq n,
$$


where $\omega=\left(\omega_{i j}\right)$ denotes an $n \times n$ matrix of sections of local 1-forms. Consequently we have

$$
\nabla_{E}(s)=\sum_{i=1}^{n} e_{i} \otimes\left(d\left(s_{i}\right)+\sum_{j=1}^{n} s_{j} \omega_{i j}\right) \equiv(d+\omega)(s) .
$$

Thus, every connection $\nabla_{E}$, where $E$ is a locally free finite rank- $n$ sheaf of modules $E$ on $X$, can be decomposed locally as follows:

$$
\nabla_{E}=d+\omega
$$

In this context, $\nabla_{E}$ is identified as a covariant derivative, being decomposed locally as a sum consisting of a flat part tautosemous with $d$, and a generally nonflat part $\omega$, called the gauge potential (vector potential), signifying a measure of deviation from the maximally undisturbed process of dynamical variation (represented by the flat part), caused by the corresponding physical field. The behavior of the gauge potential part $\omega$ of $\nabla_{E}$ under local gauge transformations constitutes the "transformation law of vector potentials" and is established in the following manner: Let $e^{U} \equiv\left\{U ; e_{i=1 \cdots n}\right\}$ and $h^{V} \equiv\left\{V ; h_{i=1 \cdots n}\right\}$ be two coordinatizing open covers of $E$ over the open sets $U$ and $V$ of $X$, such that $U \cap V \neq \emptyset$. Let us denote by $g=\left(g_{i j}\right)$ the following change of local gauge matrix:

$$
h_{j}=\sum_{i=1}^{n} g_{i j} e_{i}
$$

Under such a local gauge transformation $\left(g_{i j}\right)$, the gauge potential part $\omega$ of $\nabla_{E}$ transforms as follows:

$$
\omega^{\prime}=g^{-1} \omega g+g^{-1} d g
$$

Furthermore, it is instructive to find the local form of the curvature $\mathbf{R}_{\nabla}$ of a connection $\nabla_{E}$, where $E$ is a locally free finite rank- $n$ sheaf of modules $E$ on $X$, defined by the following A-linear morphism of sheaves:

$$
\mathbf{R}_{\nabla}:=\nabla^{1} \circ \nabla^{0}: E \longrightarrow \Omega^{2}(\mathbb{A}) \underset{\mathbb{A}}{\bigotimes} E:=\Omega^{2}(E)
$$

Due to its property of $\mathbb{A}$-covariance, a nonvanishing curvature represents, in this context, the $\mathbb{A}$-covariant and thus, observable (by $\mathbb{A}$-scalars) disturbance from the maximally symmetric state of the variation caused by the corresponding physical field. In this sense, it may be accurately characterized physically as "gauge field strength". Moreover, since the curvature $\mathbf{R}_{\nabla}$ is an $\mathbb{A}$-linear morphism of sheaves of $\mathbb{A}$-modules, $\mathbf{R}_{\nabla}$ may be thought of as an element of $\operatorname{End}(E) \otimes_{\mathbb{A}} \Omega^{2}(\mathbb{A}):=\Omega^{2}(\operatorname{End}(E))$, that is,

$$
\mathbf{R}_{\nabla} \in \Omega^{2}(\operatorname{End}(E))
$$


Hence, the local form of the curvature $\mathbf{R}_{\nabla}$ of a connection $\nabla_{E}$ consists of local $n \times n$ matrices having for entries local 2-forms on $X$.

The behavior of the curvature $\mathbf{R}_{\nabla}$ of a connection $\nabla_{E}$ under local gauge transformations constitutes the "transformation law of gauge field strengths." If we agree that $g=\left(g_{i j}\right)$ denotes the change of gauge matrix - we have previously considered in the discussion of the transformation law of gauge potentials-we deduce the following local transformation law of gauge field strengths:

$$
\mathbf{R}_{\nabla} \stackrel{g}{\longmapsto} \mathbf{R}_{\nabla^{\prime}}=g^{-1}\left(\mathbf{R}_{\nabla}\right) g
$$

According to the above dynamical framework, applications of ADG include the reformulation of Gauge theories in sheaf-theoretic terms $[15,16]$, as well as the pertinent study of the problem of manifold singularities appearing in the context of general relativity $[3,4,17-21]$. Related with the first issue, ADG has modeled Yang-Mills fields in terms of appropriate pairs $\left(E, \Phi_{\mathrm{E}}\right)$, where $E$ are vector sheaves whose sections have been identified with the states of the corresponding particles, and $\Phi_{E}$ are connections that act on the corresponding states causing interactions by means of the respective fields they refer to. Related with the second issue, ADG has replaced the sheaf of $\mathbb{R}$-algebras $\mathcal{C}^{\infty}(M)$ of smooth real-valued functions on a differential manifold with a sheaf of $\mathbb{R}$-algebras that incorporates the singularities of the manifold in terms of appropriate ideals, allowing the formulation of Einstein's equations in a covariant form with respect to the generalized scalars of that sheaf of $\mathbb{R}$-algebras. An overview of the didactics of topological sheafification of field dynamics according to ADG has been recently presented in [22], whereas the general underlying philosophical framework of ADG, pointing towards the general conception and implementation of relational mathematics, has been recently summarized in [23, 24].

\section{Quantum Localization of the Kähler-de Rham Differential Mechanism}

The basic defining feature of the quantum theory according to the Bohrian interpretation [2527], in contradistinction to all classical theories, is the realization that physical observables are not definitely or sharply valued as long as a measurement has not taken place, meaning both the precise specification of a concrete experimental context and also the registration of a value of a measured quantity in that context by means of an apparatus. Furthermore, Heisenberg's uncertainty relations determine the limits for simultaneous measurements of certain pairs of complementary physical properties, like position and momentum. In a welldefined sense, the uncertainty relations may be interpreted as measures of the valuation vagueness associated with the simultaneous determination of all physical observables in the same experimental context. In all classical theories, the valuation algebra is fixed once and for all to be the algebra of real numbers $R$, reflecting the fact that values admissible as measured results must be real numbers, irrespective of the measurement context and simultaneously for all physical observables.

The resolution of valuation vagueness in the quantum theory can be algebraically comprehended through the notion of local relativization of representability of the valuation algebra with respect to commutative algebraic contexts that correspond to prepared measurement environments [7-12]. Only after such a relativization, the eigenvalue equations formulated in the context of such a measurement environment yield numbers corresponding 
to measurement outcomes. At a logical level, commutative contexts of measurement correspond to Boolean algebras, identified as subalgebras of a quantum logical observable algebra. In the general case, commutative algebraic contexts are identified with commutative $\mathcal{R}$ algebras, which may be thought of as subalgebras of a globally noncommutative algebra of quantum observables represented irreducibly as an algebra of hypermaximal Hermitian operators on a complex Hilbert space of quantum states [10].

The decisive fact that implies the relativization of quantum representability of the valuation algebra with respect to commutative algebraic contexts is due to the observation that a globally noncommutative or even partially commutative algebra of quantum observables determines an underlying categorical diagram (presheaf) of commutative observable algebras. Then, each one of the latter can be locally identified with a commutative algebra of classical observables. Consequently, the physical information contained in a quantum observable algebra can be recovered by a gluing sheaf-theoretic construction referring to its local commutative subalgebras [10]. According to this idea, the representation of a quantum observable algebra $A_{Q}$, in the category $\mathcal{A}_{Q}$, is effected by means of the functor of points of a quantum observable algebra, restricted to all these morphisms in $\mathcal{A}_{Q}{ }^{\mathrm{op}}$ having as domains spaces (maximal ideal spectra) corresponding to commutative subalgebras of a quantum observable algebra. In this way, the variation of generalized points over all domain objects of the subcategory of $\mathscr{A}_{Q}$ op consisting of commutative algebras of observables produces the functor of points of $A_{Q}$ restricted to the subcategory of commutative objects, identified with $A_{C}{ }^{\text {op }}$. This functor of points of $A_{Q}$ is made then an object in the category of presheaves Sets $^{A C} C^{\text {op }}$, representing a quantum observables algebra in the environment of the topos of presheaves over the category of its commutative subalgebras.

More precisely, we make the basic assumption that there exists a coordinatization functor, $\mathbf{M}: \mathscr{A}_{C} \rightarrow \mathscr{A}_{Q}$, which assigns to commutative observable algebras in $\mathscr{A}_{C}$, that instantiates a model category, the underlying quantum algebras from $A_{Q}$ and to commutative algebras morphisms the underlying quantum algebraic morphisms. If $A_{C}{ }^{\text {op }}$ is the opposite category of $A_{C}$, then Sets ${ }^{A_{C}}{ }^{\text {p }}$ denotes the functor category of presheaves of commutative observable algebras, which has objects all functors $\mathbf{P}: \mathscr{A}_{C}{ }^{\text {op }} \rightarrow$ Sets and morphisms all natural transformations between such functors. Each object $\mathbf{P}$ in this category is a contravariant set-valued functor on $A_{C}$, called a presheaf on $\mathcal{A}_{C}$. The functor category of presheaveson commutative observable algebras Sets ${ }^{\mathcal{A}}{ }^{\mathrm{op}}$, exemplifies a well-defined notion of a universe of variable sets, and is characterized as a topos of presheaves. Now, if we consider the category of quantum observables algebras $\mathcal{A}_{Q}$ and the coordinatization functor $\mathbf{M}$, we can define the functor

$$
\mathrm{F}: \mathcal{A}_{Q} \longrightarrow \text { Sets }^{{ }^{A} C^{\text {op }}}
$$

from $A_{Q}$ to the category of presheaves of commutative observables algebras given by

$$
\mathbf{F}\left(A_{Q}\right)(-):=\operatorname{Hom}_{A_{Q}}\left(\mathbf{M}(-), A_{Q}\right) .
$$

Then, the representation of a quantum observable algebra $A_{Q}$, by means of its functor of points, is based on the existence of a categorical adjunction $L \dashv \mathbf{F}$ as follows [10]:

$$
\text { L : Sets }{ }^{A C^{\mathrm{op}}} \leftrightarrows \mathcal{A}_{Q}: \mathbf{F}
$$


which says that the functor of points of a quantum observable algebra restricted to commutative observable algebras $\mathbf{F}\left(A_{Q}\right)(-)$ has a left adjoint

$$
\text { L : Sets }{ }^{A_{C} \mathrm{op}} \longrightarrow \mathcal{A}_{Q}
$$

which is defined for each presheaf $\mathbf{P}$ in Sets ${ }^{A} c^{\text {op }}$ as the colimit

$$
\mathbf{L}(\mathbf{P})=\underset{A_{C}}{\mathbf{P}} \mathbb{M}
$$

Equivalently, there exists a bijection, natural in $\mathbf{P}$ and $A_{Q}$ as follows:

$$
\operatorname{Nat}\left(\mathbf{P}, \mathbf{F}\left(A_{Q}\right)\right) \cong \operatorname{Hom}_{A_{Q}}\left(\mathbf{L P}, A_{Q}\right) .
$$

As a consequence, we depict the category of presheaves $\operatorname{PShv}\left(A_{C}\right):=\operatorname{Sets}^{A_{C}{ }^{o p}}$ as the appropriate topos for the generation of a quantum dynamical mechanism. Furthermore, a suitable localization scheme applicable to the quantum regime of observable structure is obtained by the notion of a Grothendieck topology, defined in terms of covers on the base category $A_{C}$. More concretely, a Grothendieck topology suitable for this purpose is defined by means of a covering system $S$ of epimorphic families on the base category of commutative contexts, by requiring that the morphism

$$
G_{S}: \coprod_{\left\{s: A_{\left.C^{\prime} \rightarrow A_{C}\right\} \in S}\right.} A_{C}{ }^{\prime} \longrightarrow A_{C}
$$

where $A_{C}, A_{C}{ }^{\prime}$ in $A_{C}$, is an epimorphism in $\mathcal{A}_{Q}$ [10]. In this way, the Grothendieck topology defined above specifies functorially a physical localization scheme suited for probing the quantum regime of observable structure.

Now, it is important to notice that the counit of the adjunction

$$
\begin{aligned}
& \text { L : Sets }{ }^{A^{\circ p}} \leftrightarrows \mathcal{A}_{Q}: \text { F } \\
& \varepsilon_{A_{Q}}: \mathbf{L F}\left(A_{Q}\right) \longrightarrow A_{Q},
\end{aligned}
$$

defined by the composite endofunctor

$$
\mathrm{G}:=\mathrm{LF}: \mathscr{A}_{Q} \longrightarrow \mathcal{A}_{Q}
$$

constitutes the first step of a functorial free resolution of a quantum observable algebra $A_{Q}$ in $A_{Q}$. Actually, by iterating the endofunctor $\mathrm{G}$, we may extend $\varepsilon_{A_{Q}}$ to a free simplicial resolution of $A_{Q}$. In this setting, we may now apply Kähler's methodology in order to obtain the object of quantum differential 1-forms, by means of the following split short exact sequence:

$$
0 \longrightarrow \Omega_{A_{Q}} \longrightarrow \mathrm{G} A_{Q} \longrightarrow A_{Q}
$$


or equivalently

$$
0 \longrightarrow \Omega_{A_{Q}} \longrightarrow \mathbf{F}\left(A_{Q}\right) \underset{A_{C}}{\otimes} \mathbf{M} \longrightarrow A_{Q}
$$

According to the above, we obtain that

$$
\Omega_{A_{Q}}=\frac{J}{J^{2}}
$$

where $J=\operatorname{Ker}\left(\varepsilon_{A_{Q}}\right)$ denotes the kernel of the counit of the adjunction. At a next stage, we notice that the functor of points of a quantum observable algebra restricted to commutative algebras $\mathbf{F}\left(A_{Q}\right)$ is left exact, because it is the right adjoint functor of the established adjunction. Thus, it preserves the short exact sequence defining the object of quantum differential 1-forms, in the following form:

$$
0 \longrightarrow \mathbf{F}\left(\Omega_{A_{Q}}\right) \longrightarrow \mathbf{F}\left(\mathbf{G}\left(A_{Q}\right)\right) \longrightarrow \mathbf{F}\left(A_{Q}\right)
$$

Hence, we immediately obtain that

$$
\mathbf{F}\left(\Omega_{A_{Q}}\right)=\frac{Z}{Z^{2}}
$$

where $Z=\operatorname{Ker}\left(\mathbf{F}\left(\varepsilon_{A_{Q}}\right)\right)$. Then, by analogy to the general algebraic situation, interpreted inside the proper universe that the functor of points of a quantum observable algebra assumes existence, namely, the topos Sets ${ }^{A} C^{\mathrm{op}}$, we introduce the notion of an interaction field, termed quantum field, by means of the functorial pair $\left(\mathbf{F}\left(A_{Q}\right):=\operatorname{Hom}_{A_{Q}}\left(\mathbf{M}(-), A_{Q}\right), \nabla_{\mathbf{F}\left(A_{Q}\right)}\right)$, where the quantum connection $\nabla_{\mathbf{F}\left(A_{Q}\right)}$ is defined as the following natural transformation:

$$
\nabla_{\mathbf{F}\left(A_{Q}\right)}: \mathbf{F}\left(A_{Q}\right) \longrightarrow \mathbf{F}\left(\Omega_{A_{Q}}\right)
$$

Thus, the quantum connection $\nabla_{\mathbf{F}\left(A_{Q}\right)}$ induces a sequence of functorial morphisms or equivalently natural transformations as follows:

$$
\mathbf{F}\left(A_{Q}\right) \longrightarrow \mathbf{F}\left(\Omega_{A_{Q}}\right) \longrightarrow \cdots \longrightarrow \mathbf{F}\left(\Omega^{n}{ }_{A_{Q}}\right) \longrightarrow \cdots
$$

Let us denote by

$$
\mathbf{R}_{\nabla}: \mathbf{F}\left(A_{Q}\right) \longrightarrow \mathbf{F}\left(\Omega^{2}{ }_{A_{Q}}\right)
$$

the composition $\nabla^{1} \circ \nabla^{0}$ in the obvious notation, where $\nabla^{0}:=\nabla_{\mathbf{F}\left(A_{Q}\right)}$, which we call the curvature of the quantum connection $\nabla_{\mathbf{F}\left(A_{Q}\right)}$. The latter sequence of functorial morphisms is actually a complex if and only if $\mathbf{R}_{\nabla}=0$. We say that the quantum connection $\nabla_{\mathbf{F}\left(A_{Q}\right)}$ is integrable or flat if $\mathbf{R}_{\nabla}=0$, referring to the above complex as the functorial de Rham 
complex of the integrable connection $\nabla_{\mathrm{F}\left(A_{Q}\right)}$ in that case. The vanishing of the curvature of the quantum connection, that is,

$$
\mathbf{R}_{\nabla}=0
$$

can be used as a means of transcription of Einstein's equations in the quantum regime, in the absence of cohomological obstructions. We may explain the curvature of the quantum connection as the effect of nontrivial interlocking of the information carried by commutative reference contexts, in some underlying diagram of a quantum algebra of observables, being formed by such localizing commutative observables subalgebras. The nontrivial gluing of the information of commutative contexts in localization systems of a quantum algebra is caused by topological obstructions that, in turn, are being co-implied with respect to the nontriviality of the cohomology groups of the corresponding algebraic de Rham complex. Intuitively, a nonvanishing curvature is the nonlocal attribute detected by an observer employing a commutative reference context in a discretely topologized categorical environment, in the attempt to understand the quantum localization properties, after having introduced a potential (quantum gravitational connection) in order to account for the latter by means of a differential geometric mechanism. Thus, the physical meaning of curvature is associated with the apparent existence of nonlocal correlations from the restricted spatial perspective of disjoint classical commutative contexts $A_{C}$. It is instructive to make clear that, in the present schema, the notion of curvature does not refer to an underlying background manifold, since such a structure has not been required at all in the development of the differential geometric mechanism, according to functorial algebraic methods.

\section{Conclusions}

Conclusively, it is worthwhile to emphasize that discussions, of background manifold independence pertaining to the current research focus in quantum gravity, should take at face value the fact that the fixed manifold construct in general relativity is just the byproduct of fixing physical representability in terms of the field of real numbers. Moreover, it is completely independent of the possibility of formulating dynamics, since the latter can be developed precisely along purely algebraic lines, that is, by means of functorial connections. Hence, the usual analytic differential geometric framework of smooth manifolds, needed for the formulation of classical General Relativity, is just a special coordinatization of the universal functorial mechanism of infinitesimal scalars extension and thus should be substituted appropriately, in case a merging with quantum theory is sought. The substitution is guided by the principle of relativized representability, which forces the topos Sets ${ }^{A} c^{\text {op }}$ as the proper universe of discourse for probing the quantum regime. In turn, this requires a functorial adaptation of the algebraic mechanism of connections inside this topos, and subsequently, an interpretation of quantum gravitational dynamics sheaf cohomologically with respect to the nontrivial localization schemes of observables in the quantum regime. The central conceptual issue characterizing the present sheaf-theoretic approach, developed on the basis of the general algebraic homological framework of functorial dynamics constructed in [1], resolves in the explicit consideration and intrinsic modeling of generalized localization environments for the formulation of physical interaction processes. More concretely, in classical theories, localization has been conceived by means of metrical properties on a preexisting smooth set-theoretic spacetime manifold. In contradistinction, quantum 
localization should be understood categorically and sheaf-theoretically, that is purely in functorial terms of relational information between quantum observable algebras and covering diagrams of commutative ones, without any supporting notion of a fixed smooth background manifold.

\section{References}

[1] A. Mallios and E. Zafiris, "The homological Kähler-de Rham differential mechanism-part I: application in general theory of relativity," Advances in Mathematical Physics, vol. 2011, Article ID 191083, 14 pages, 2011.

[2] A. Mallios, "On localizing topological algebras," in Topological Algebras and Their Applications, vol. 341, pp. 79-95, American Mathematical Society, Providence, RI, USA, 2004.

[3] A. Mallios and E. E. Rosinger, "Abstract differential geometry, differential algebras of generalized functions, and de Rham cohomology," Acta Applicandae Mathematicae, vol. 55, no. 3, pp. 231-250, 1999.

[4] A. Mallios and E. E. Rosinger, "Space-time foam dense singularities and de Rham cohomology," Acta Applicandae Mathematicae, vol. 67, no. 1, pp. 59-89, 2001.

[5] S. MacLane, Categories for the Working Mathematician, Springer, New York, NY, USA, 1971.

[6] S. MacLane and I. Moerdijk, Sheaves in Geometry and Logic, Springer, New York, NY, USA, 1992.

[7] E. Zafiris, "Generalized topological covering systems on quantum events' structures," Journal of Physics. A, vol. 39, no. 6, pp. 1485-1505, 2006.

[8] E. Zafiris, "Probing quantum structure with Boolean localization systems," International Journal of Theoretical Physics, vol. 39, no. 12, pp. 2761-2778, 2000.

[9] E. Zafiris, "Boolean coverings of quantum observable structure: a setting for an abstract differential geometric mechanism," Journal of Geometry and Physics, vol. 50, no. 1-4, pp. 99-114, 2004.

[10] E. Zafiris, "Quantum observables algebras and abstract differential geometry: the topos-theoretic dynamics of diagrams of commutative algebraic localizations," International Journal of Theoretical Physics, vol. 46, no. 2, pp. 319-382, 2007.

[11] E. Zafiris, "Quantum event structures from the perspective of Grothendieck topoi," Foundations of Physics, vol. 34, no. 7, pp. 1063-1090, 2004.

[12] E. Zafiris, "Interpreting observables in a quantum world from the categorial standpoint," International Journal of Theoretical Physics, vol. 43, no. 1, pp. 265-298, 2004.

[13] J. L. Bell, Toposes and Local Set Theories, vol. 14, The Clarendon Press, New York, NY, USA, 1988.

[14] A. Mallios, Geometry of Vector Sheaves: An Axiomatic Approach to Differential Geometry, vol. 1-2, Kluwer Academic, Dodrecht, The Netherlands, 1998.

[15] A. Mallios, Modern Differential Geometry in Gauge Theories. Vol. I, Birkhäuser, Boston, Mass, USA, 2006.

[16] A. Mallios, Modern Differential Geometry in Gauge Theories. Vol. II, Birkhäuser, Boston, Mass, USA, 2010.

[17] A. Mallios, "Remarks on "singularities"," in Progress in Mathematical Physics, A. Mallios, Ed., Nova Science, Hauppauge, NY, USA, 2003.

[18] A. Mallios, "Quantum gravity and "singularities"," Note di Matematica, vol. 25, no. 2, pp. 57-76, 2005.

[19] A. Mallios, "Geometry and physics today," International Journal of Theoretical Physics, vol. 45, no. 8, pp. 1557-1593, 2006.

[20] A. Mallios and I. Raptis, "Finitary Čech-de Rham cohomology: much ado without $C^{\infty}$-smoothness,," International Journal of Theoretical Physics, vol. 41, no. 10, pp. 1857-1902, 2002.

[21] A. Mallios and I. Raptis, " $C^{\infty}$-smooth singularities exposed: chimeras of the differential spacetime manifold," http://arxiv.org/abs/gr-qc/0411121v14. In press.

[22] I. Raptis, "A dodecalogue of basic didactics from applications of abstract differential geometry to quantum gravity," International Journal of Theoretical Physics, vol. 46, no. 12, pp. 3009-3021, 2007.

[23] A. Mallios, "On algebra spaces," in Topological Algebras and Applications, vol. 427 of Contemporary Mathematics, pp. 263-283, American Mathematical Society, Providence, RI, USA, 2007.

[24] A. Mallios, "A-invariance: an axiomatic approach to quantum relativity," International Journal of Theoretical Physics, vol. 47, no. 7, pp. 1929-1948, 2008.

[25] N. Bohr, Atomic Physics and Human Knowledge, John Wiley, New York, NY, USA, 1958.

[26] H. J. Folse, The Philosophy of Niels Bohr. The Framework of Complementarity, North Holland, New York, NY, USA, 1985.

[27] J. Bub, Interpreting the quantum world, Cambridge University Press, Cambridge, 1997. 


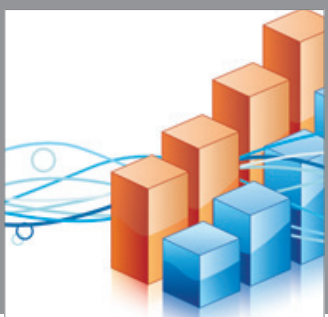

Advances in

Operations Research

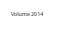

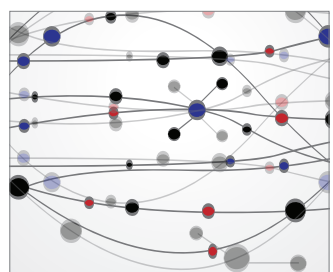

\section{The Scientific} World Journal
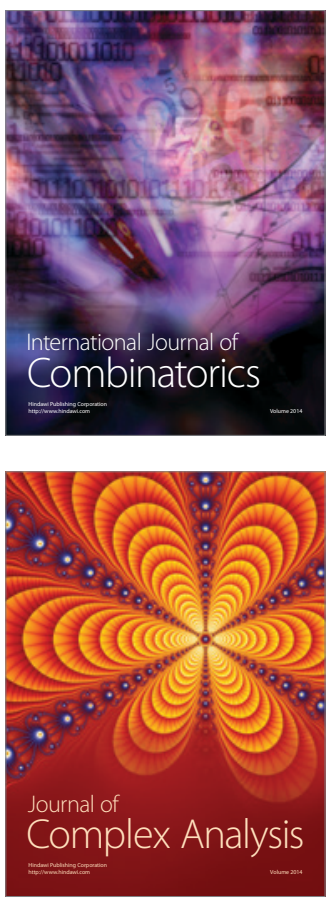

International Journal of

Mathematics and

Mathematical

Sciences
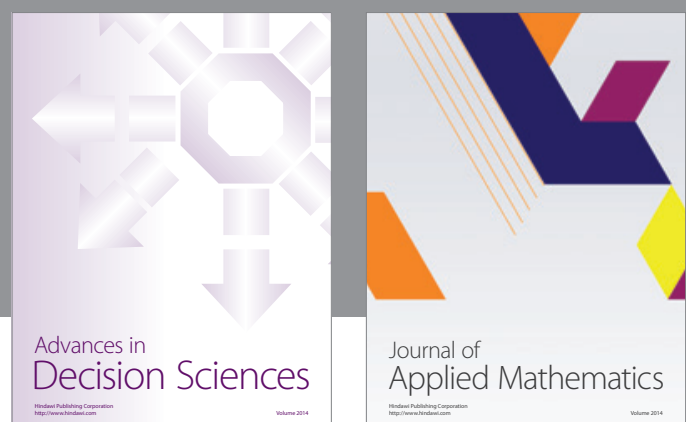

Journal of

Applied Mathematics
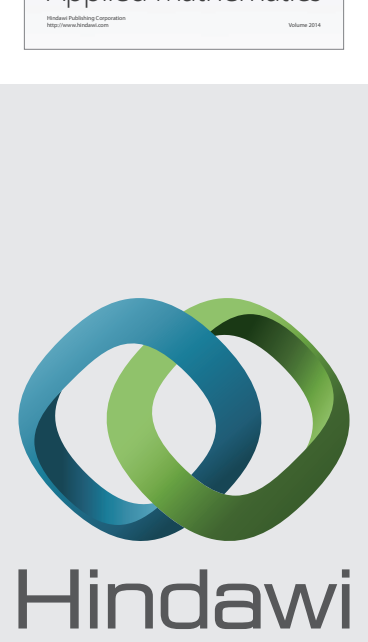

Submit your manuscripts at http://www.hindawi.com
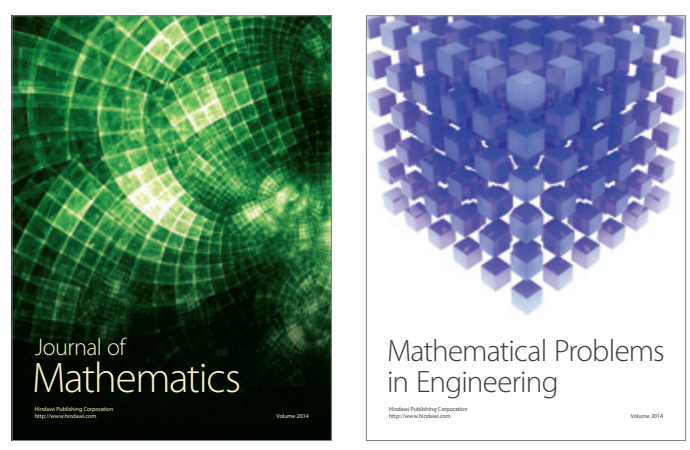

Mathematical Problems in Engineering
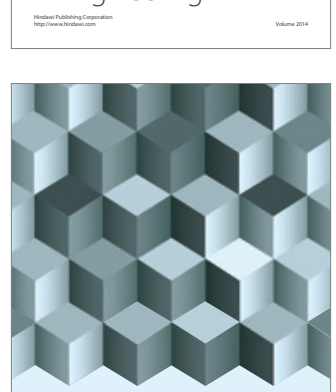

Journal of

Function Spaces
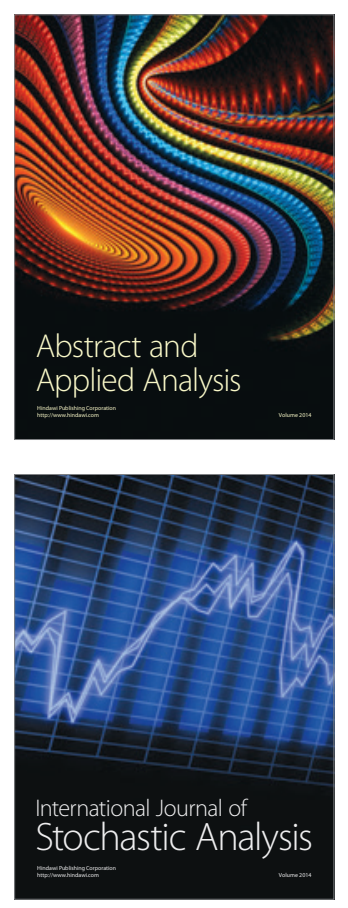

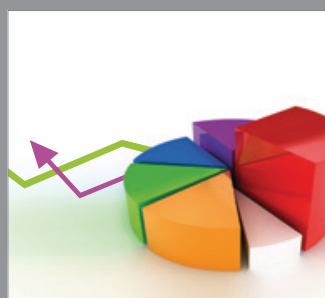

ournal of

Probability and Statistics

Promensencen
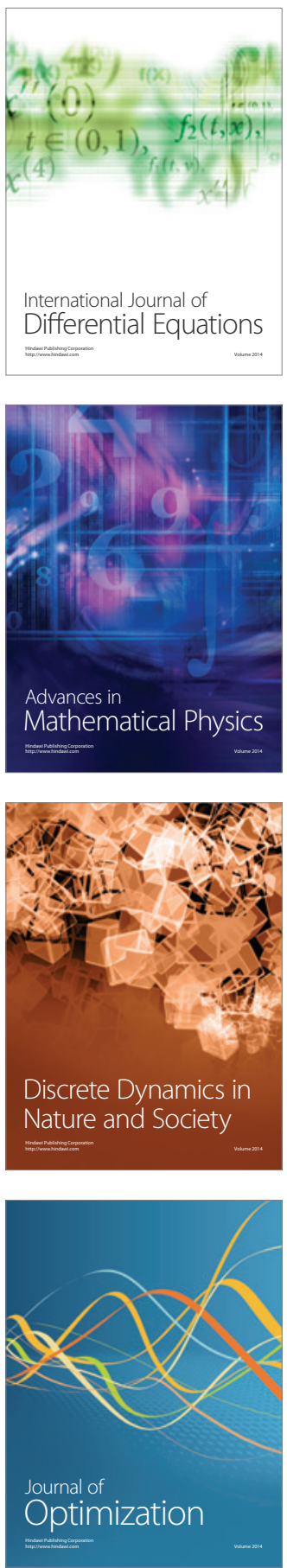